Case Report

\title{
A case report of Dengue Encephalitis
}

\author{
WAPSR Weerarathna ${ }^{1}$, T Peranatharajha ${ }^{1}$ \\ Sri Lankan Journal of Infectious Diseases 2016 Vol.6 (1):51-54 \\ DOI: http://dx.doi.org/10.4038/sljid.v6i1.8081
}

\begin{abstract}
Dengue fever is a common mosquito borne viral febrile illness which rarely affects the central nervous system and presents with encephalitis. This case is about a 14 years old boy who presented with high grade fever and generalized tonic-clonic seizures. Electroencephalogram showed generalized spike activity and periodicity with normal CT scan of the brain. Dengue encephalitis was diagnosed with a positive Dengue IgM in CSF on the sixth day of illness. He was discharged 8 days after admission without any neurological deficit. This case highlights the importance of maintaining a high index of suspicion in arriving at the correct diagnosis in rare presentations of common diseases.
\end{abstract}

Keywords: Dengue, dengue encephalitis

\section{Introduction}

Dengue fever is a common mosquito borne viral febrile illness which has varying clinical presentations, ranging from asymptomatic infection to potentially life threatening dengue haemorragic fever (DHF) and dengue shock syndrome (DSS). ${ }^{1}$ It is known to cause central nervous system (CNS) manifestations due to neurovirulent properties of serotypes 2 and 3, but encephalitis is a rarity. ${ }^{2,3}$ Isolation of the virus or the detection of dengue genome from the brain tissue or cerebrospinal fluid (CSF) confirms the diagnosis and in the absence of confirmatory tests, IgM in the CSF is supportive of the diagnosis. ${ }^{4,5}$ We report a case of a young boy who presented with fever and convulsions in whom a diagnosis of dengue encephalitis was made.

\section{Case report}

A 14 year old boy from Jaffna presented with high fever for 3 days and generalized tonic-clonic seizures lasting a few minutes. On admission he was in post ictal drowsiness with no features of meningism. He was neither pale nor icteric but diaphoretic with a temperature of $38.9{ }^{\circ} \mathrm{C}$ and normal vital functions. There were no rashes and papilloedema was not evident on funduscopy. Focused history revealed no past history of epilepsy or other illnesses. CNS examination revealed no focal neurological deficits and the rest of the systemic examination was unremarkable.

${ }^{1}$ Teaching Hospital Jaffna, Sri Lanka

Address for correspondence: Dr W.A.P.S.R.Weerarathna; Registrar in Medicine, Teaching Hospital Jaffna, Sri Lanka, Telephone:+94774436136 E-mail: sunethweerarathna@ gmail.com

Received 20 April 2015 and revised version accepted 18 February 2016 
On day 3 of fever, his full blood count results were Hb 12.9 g/dl, PCV 37.9\%, WBC 3.3x $10^{3} \mathrm{~mm}^{3}$ and platelet count $110 \times 10^{3} \mathrm{~mm}^{3}$. His ESR was $12 \mathrm{~mm} / \mathrm{hr}$ and blood sugar, liver and renal functions including serum electrolytes were unremarkable. Dengue virus NS1 Antigen (ELISA) was positive.

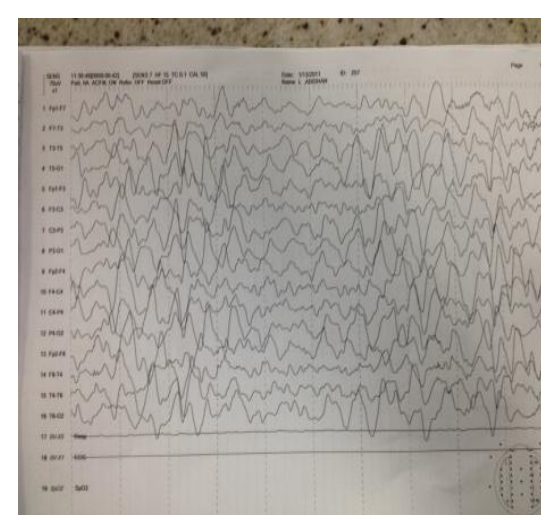

Figure 1: EEG showing features of encephalitis.

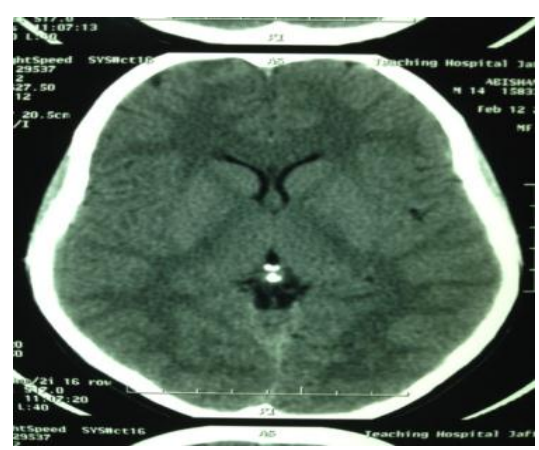

Figure 2: Non contrast CT scan of the head.
EEG was reported as generalized spike activity and periodicity showing encephalitis (Figure 1). Non contrast CT scan of the head was normal (Figure 2). His vital functions including urine output were closely monitored in the Medical Intensive Care Unit (MICU) and managed according to ICU protocols. Oral phenytoin sodium $150 \mathrm{mg}$ bd and intravenous cefotaxime $1 \mathrm{~g}$ tds were started empirically on a clinical diagnosis of meningoencephalitis. At this juncture he had fever. However, there were no recurrent seizures. On day 6 of illness, dengue antibodies Ig $\mathrm{M}$ and IgG were reported as positive (ELISA method). FBC showed $\mathrm{Hb}$ of $13.4 \mathrm{~g} / \mathrm{dl}$, PCV $40 \%$, WBC $2.8 \times 10^{3} \mathrm{~mm}^{3}$ and platelet count of $76 \times 10^{3} \mathrm{~mm}^{3}$. SGOT and SGPT levels were $98 \mathrm{iu} / 1$ and $80 \mathrm{iu} / 1$ respectively. INR and APTT levels were normal. Urinalysis and blood cultures were found to be negative.

CSF analysis on day 6 showed clear fluid, protein 28.9 $\mathrm{mg} / \mathrm{dl}$, sugar $73 \mathrm{mg} / \mathrm{dl}$, (random blood sugar $110 \mathrm{mg} / \mathrm{dl}$ ), no lymphocytes, polymorphs or red cells. CSF for dengue IgM was reported as positive with negative IgG (ELISA). CSF for JE and HSV Ig M by ELISA method were negative. PCR for Herpes simplex and dengue viruses were not available at the time for confirmatory testing. Virus typing was not performed due to lack of facilities.

On day 7 of illness the patient's fever subsided and he was found to have mild pleural effusion on the right side. No ascites was detected. He was haemodynamically stable without any haemorrhagic diathesis. Ultrasound scan showed minimal pleural effusion on the right side with a thin rim of fluid in the hepatorenal pouch. Chest radiograph was unremarkable.

On day 10 of illness he made a good recovery without any neurological deficits or residual effects. On discharge his platelet count was $170 \times 10^{3} \mathrm{~mm}^{3}$. Intravenous antibiotics were continued for 5 days and Phenytoin sodium was continued. The patient was diagnosed with DHF with the aid of clinical and laboratory findings and the case was notified.

\section{Discussion:}

Dengue fever is caused by a flavivirus with four serotypes, DEN-1 to DEN-4. It is endemic in the tropics and subtropics and transmitted by the Aedes mosquito. Neurological features were not thought to be prominent in dengue illness until recently, when they were noted in prospective 
studies on patients with dengue and encephalitis in various cohorts in South East Asia, India and South America. ${ }^{6}$

Neurological manifestations of dengue fever are known to occur with encephalopathy, encephalitis, neuromuscular involvement such as Guillain-Barre syndrome, polyneuropathy or transverse myelitis and neuro-ophthalmic involvement. Dengue encephalopathy and dengue encephalitis may be difficult to distinguish clinically as both may present with clouding of consciousness. Dengue encephalopathy is due to metabolic disorders including hyponatraemia, hepatic failure, hypotension, cerebral edema, disseminated intravascular coagulation or frank haemorrhage. Encephalitis is attributed to infiltration of virus infected macrophages into the brain $^{1}$ and dengue virus type $2 \& 3$ have been commonly demonstrated in the CSF of patients with dengue encephalitis. ${ }^{2}$ Direct viral invasion during the viraemic phase and virus entry when the integrity of blood brain barrier is transiently compromised during infection is also suggested as means of entry. ${ }^{9}$

This patient had serologically confirmed dengue fever and ultrasound demonstrable fluid leakage. CSF invasion by the dengue virus is suggested by a positive dengue $\operatorname{IgM}$ on day 6 of the illness with a negative IgG. EEG patterns in dengue encephalitis may include unilateral or bilateral periodic sharp waves or attenuation of amplitude, focal or generalized slow waves, epileptiform discharges or electrical seizures. This patient had generalized spike activity and periodicity. The outcome of dengue encephalitis may be an uneventful recovery ${ }^{1}$, neurological deficits or death. ${ }^{4}$ Treatment is symptomatic and supportive. We managed the patient symptomatically and he made an uneventful recovery.

\section{Conclusion}

Dengue fever is a prevalent infection in Sri Lanka at the moment causing high morbidity and a considerable burden on the health budget of the country. It is important to be aware of and detect uncommon presentations of dengue. Reporting such presentations is therefore important. A literature search on dengue encephalitis in the Jaffna district yielded negative results suggesting that this is the first reported case of dengue encephalitis in this district.

\section{Competing interests}

The authors declare that they have no competing interests.

\section{Acknowledgements}

We would like to express our deepest gratitude to this patient for allowing us to report this case and to the Medical Research Institute (MRI), Colombo, for providing investigation results.

\section{References}

1. Koley TK., Jain S., Sharma H., et al. Dengue encephalitis. J Assoc Physicians India 2003; 51: 422-3. No doi

2. Lum LCS., Lam SK., Choy YS., et al. Dengue encephalitis: A true entity? Am J Trop Med Hyg 1996; 54:256-9. No doi 
3. Lum LCS., Lam SK., George R., et al. Fulminant hepatitis in dengue infection. Southeast Asian J Trop Med Public Health 1993; 24:467-71 No doi

4. Kankirawatana P., Chokephaibulkit K., Puthavathana P., et al. Dengue infection presenting with central nervous system manifestation. J Child Neurol 2000; 15:544-7. http://dx.doi.org/10.1177/088307380001500809

5. Pancharoen C., Thisyakorn U. Neurological manifestations in dengue patients. Southeast Asian J Trop Med Public Health 2001; 32:341-5. No doi

6. Carod-Artal FJ., Wichmann O., Farrar J., Gascón J. Neurological complications of dengue virus infection. Lancet Neurol 2013; 12(9):906-19. http://dx.doi.org/10.1016/S1474-4422(13)70150-9

7. Ramos C., Sánchez G., Pando RH., et al. Dengue virus in the brain of a fatal case of hemorrhagic dengue fever. Journal of Neurovirology 1998; 4(4):465-8. http://dx.doi.org/10.3109/13550289809114548 